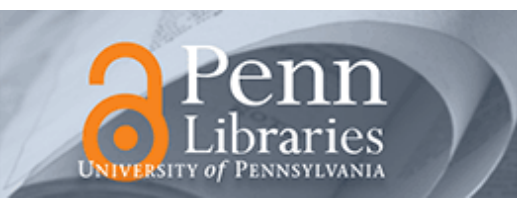

University of Pennsylvania

ScholarlyCommons

4-2017

\title{
MOTIVATional intErviewing to Improve Self-Care in Heart Failure Patients (MOTIVATE-HF): Study Protocol of a Three-Arm Multicenter Randomized Controlled Trial
}

Ercole Vellone

Marco Paturzo

Fabio D'Agostino

Antonio Petruzzo

Stefano Masci

See next page for additional authors

Follow this and additional works at: https://repository.upenn.edu/nrs

Part of the Behavioral Medicine Commons, Cardiology Commons, Cardiovascular Diseases

Commons, Circulatory and Respiratory Physiology Commons, Medical Humanities Commons, Nursing

Commons, and the Preventive Medicine Commons

\section{Recommended Citation}

Vellone, E., Paturzo, M., D'Agostino, F., Petruzzo, A., Masci, S., Ausili, D., Rebora, P., Alvaro, R., \& Riegel, B. (2017). MOTIVATional intErviewing to Improve Self-Care in Heart Failure Patients (MOTIVATE-HF): Study Protocol of a Three-Arm Multicenter Randomized Controlled Trial. Contemporary Clinical Trials, 55 34-38. http://dx.doi.org/10.1016/j.cct.2017.02.003 


\title{
MOTIVATional intErviewing to Improve Self-Care in Heart Failure Patients (MOTIVATE-HF): Study Protocol of a Three-Arm Multicenter Randomized Controlled Trial
}

\author{
Abstract \\ Aims \\ Heart Failure (HF) self-care improves patient outcomes but trials designed to improve HF self-care have \\ shown inconsistent results. Interventions may be more effective in improving self-care if they mobilize \\ support from providers, promote self-efficacy, increase understanding of HF, increase the family \\ involvement, and are individualized. All of these elements are emphasized in motivational interviewing \\ (MI); few trials have been conducted using $\mathrm{MI}$ in $\mathrm{HF}$ patients and rarely have caregivers been involved in \\ $\mathrm{Ml}$ interventions. The aim of this study will be to evaluate if Ml improves self-care maintenance in $\mathrm{HF}$ \\ patients, and to determine if $\mathrm{Ml}$ improves the following secondary outcomes: a) in HF patients: self-care \\ management, self-care confidence, symptom perception, quality of life, anxiety/depression, cognition, \\ sleep quality, mutuality with caregiver, hospitalizations, use of emergency services, and mortality; b) in \\ caregivers: caregiver contribution to self-care, quality of life, anxiety/depression, sleep, mutuality with \\ patient, preparedness, and social support. \\ Methods
}

A three-arm randomized controlled trial will be conducted in a sample of $240 \mathrm{HF}$ patients and caregivers. Patients and caregivers will be randomized to the following arms: 1) Ml intervention to patients only; 2) MI intervention to patients and caregivers; 3 ) standard of care to patients and caregivers. The primary outcome will be measured in patients 3 months after enrollment. Primary and secondary outcomes also will be evaluated 6,9 and 12 months after enrollment.

\section{Conclusion}

This study will contribute to understand if MI provided to patients and caregivers can improve self-care. Because HF is rising in prevalence, findings can be useful to reduce the burden of the disease.

\section{Keywords}

heart failure, self-care, motivational interviewing, caregivers

\section{Disciplines}

Behavioral Medicine | Cardiology | Cardiovascular Diseases | Circulatory and Respiratory Physiology | Medical Humanities | Medicine and Health Sciences | Nursing | Preventive Medicine

\section{Author(s)}

Ercole Vellone, Marco Paturzo, Fabio D'Agostino, Antonio Petruzzo, Stefano Masci, Davide Ausili, Paola Rebora, Rosaria Alvaro, and Barbara Riegel 


\author{
Ercole Vellone, $\mathrm{PhD}, \mathrm{RN}$, FESC \\ Department of Biomedicine and Prevention \\ University of Rome Tor Vergata, Rome, Italy \\ Marco Paturzo, MSN, RN \\ Department of Biomedicine and Prevention \\ University of Rome Tor Vergata, Rome, Italy \\ Fabio D'Agostino PhD, RN \\ Department of Biomedicine and Prevention \\ University of Rome Tor Vergata, Rome, Italy \\ Antonio Petruzzo MSN, RN \\ Department of Biomedicine and Prevention \\ University of Rome Tor Vergata, Rome, Italy \\ Stefano Masci, MS \\ School of Counselling \\ University of Rome Tor Vergata, Rome, Italy \\ Davide Ausili, $\mathrm{PhD}, \mathrm{RN}$ \\ Department of Medicine and Surgery, \\ University of Milan-Bicocca, Monza, Italy \\ Paola Rebora, $\mathrm{PhD}$ \\ Centre of Biostatistics for Clinical Epidemiology \\ Department of Medicine and Surgery, \\ University of Milan-Bicocca, Monza, Italy. \\ Rosaria Alvaro, MSN, RN, FESC \\ Department of Biomedicine and Prevention \\ University of Rome Tor Vergata, Rome, Italy \\ Barbara Riegel, PhD, RN, FAAN, FAHA \\ School of Nursing, \\ University of Pennsylvania, Philadelphia, Pennsylvania, USA
}

\title{
Correponding author
}

Ercole Vellone

Department of Biomedicine and Prevention

University of Rome Tor Vergata, Rome, Italy

Via Montpellier, 1

00133 Rome, Italy

Phone + 390672596802; Fax +390672596961

Email: ercole.vellone@uniroma2.it 


\begin{abstract}
Aims.Heart Failure (HF) self-care improves patient outcomes but trials designed to improve HF self-care have shown inconsistent results. Interventions may be more effective in improving selfcare if they mobilize support from providers, promote self-efficacy, increase understanding of HF, increase the family involvement, and are individualized. All of these elements are emphasized in motivational interviewing (MI); few trials have been conducted using MI in HF patients and rarely have caregivers been involved in MI interventions. The aim of this study will be to evaluate if MI improves self-care maintenance in HF patients, and to determine if MI improves the following secondary outcomes: a)in HF patients: self-care management, self-care confidence, symptom perception, quality of life, anxiety/depression, cognition, sleep quality, mutuality with caregiver, hospitalizations, use of emergency services, and mortality; b)in caregivers: caregiver contribution to self-care, quality of life, anxiety/depression, sleep, mutuality with patient, preparedness, and social support.
\end{abstract}

Methods.A three-arm randomized controlled trial will be conducted in a sample of $240 \mathrm{HF}$ patients and caregivers. Patients and caregivers will be randomized to the following arms: 1)MI intervention to patients only; 2)MI intervention to patients and caregivers; 3)standard of care to patients and caregivers. The primary outcome will be measured in patients 3 months after enrollment. Primary and secondary outcomes also will be evaluated 6,9 and 12 months after enrollment.

Conclusion. This study will contribute to understand if MI provided to patients and caregivers can improve self-care. Because HF is raising in prevalence, findings can be useful to reduce the burden of the disease.

Key words: heart failure, self-care, motivational interviewing, caregivers 


\section{Introduction}

Heart failure (HF) is a chronic condition that affects over 15 million people in Europe ${ }^{1}$ and 5.7 million people in USA. ${ }^{2}$ As the final stage of a wide variety of cardiac diseases, HF is rising in prevalence worldwide because of the aging of the population. Projections show that by $2030,46 \%$ of the population aged $\geq 18$ year will be affected by HF. ${ }^{2}$

The impact of HF on the quality of life (QOL) of patients and their families is immense. Symptom burden and depression in HF patients are comparable to that of cancer. ${ }^{3}$ Family caregivers of HF patients experience greater levels of unmet needs than other caregiver populations. $^{4}$

Self-care has been shown to improve HF patient outcomes (e.g., QOL, hospitalizations). ${ }^{5} \mathrm{HF}$ self-care was defined as "a naturalistic decision-making process that influences actions that maintain physiologic stability (maintenance), facilitate the perception of symptoms (symptom perception), and direct the management of those symptoms (management)", (p. 1). ${ }^{6}$ Self-care maintenance, symptom perception, and self-care management are mastered in sequence and prior works have already shown that self-care maintenance predicts self-care management. ${ }^{7,8}$ Self-care maintenance, symptom perception and management are influenced by confidence in the ability to perform self-care or task-specific self-efficacy in the self-care process. ${ }^{6}$ Prior work Caregiver contributions to HF self-care have been defined as the "provision of time, effort, and support in behalf of another person who needs to perform HF self-care" (p. 246). ${ }^{9}$ Although patient self-care and caregiver contributions to self-care are important to improve patients' HF outcomes, both patients and caregivers struggle and self-care has been shown to be suboptimal. ${ }^{10,11}$

Trials designed to improve HF self-care have shown inconsistent results. Some studies have found that educational interventions improve self-care ${ }^{12}$ while others have not. ${ }^{13} \mathrm{~A}$ recent systematic review with meta-analysis ${ }^{14}$ that included 20 randomized controlled trials (RCTs) with a 
total of $5624 \mathrm{HF}$ patients showed that interventions designed to improve self-care were effective in reducing mortality and hospitalization; however, the authors concluded that the mechanisms by which self-care could be improved were unclear. In fact, interventions that included standardized training of interventionists, peer contact, record keeping, or goal-setting skills appeared even less effective than interventions without these characteristics. Another recent systematic review ${ }^{15}$ of 33 studies explored the main mechanisms through which programs actually work to improve HF selfcare. Results showed that interventions may be more effective if they 1) mobilize real support from providers (e.g., establishing a good supportive relationship with patients); 2) promote self-efficacy (e.g., promoting personal confidence and hope); 3) increase insight and understanding of HF and self-care (e.g., linking symptoms to HF self-care tasks); 4) increase the involvement of other people (e.g., including a family caregiver); 5) are individualized and responsive (e.g., basing the intervention on patient need and preferences). All of these mechanistic elements are emphasized in motivational interviewing.

Motivational interviewing (MI) is a counselling technique defined as a "person-centered method of guiding to elicit and strengthen personal motivation for change"16 (p. 25) with a collaborative and evocative approach that honors patient autonomy to elicit his/her own motivation to change behaviors in the interest of health. In fact, MI is described as the polar-opposite of giving unsolicited advice. ${ }^{16} \mathrm{MI}$ explores and resolves the ambivalence in individuals' behaviors (e.g., someone who considers exercise to be important but does not exercise), thereby enhancing intrinsic motivation to change. MI is based on the following principles: develop discrepancy, express empathy, avoid arguing and direct confrontation, roll with resistance, and support self-efficacy and optimism.

MI was originally developed in psychology but the technique has been adopted by healthcare providers. Several RCTs have been conducted showing that MI improves medication adherence ${ }^{17}$, dietary adherence and weight loss in diabetes patients ${ }^{18}$, and smoking cessation, depression, and quality of life in people with cardiovascular diseases. ${ }^{19}$ 
In HF care, few studies have been conducted using MI. Masterson Creber at al ${ }^{20}$ showed in a sample of 67 HF patients that those randomized in a tailored MI intervention had better self-care maintenance than those in the control group after adjusting for confounding factors. In the same sample, Riegel et $\mathrm{al}^{21}$ showed that those receiving the MI intervention had significantly fewer hospital readmissions at the three-month follow-up. Paradis et al ${ }^{22}$ in a RCT involving $30 \mathrm{HF}$ patients showed that MI improved self-care confidence significantly and Brodie et $\mathrm{al}^{23}$ showed that MI improved generic and disease-specific QOL in HF patients. Finally, a mixed method study conducted by Riegel et al ${ }^{24}$ on 41 HF patients provided qualitative evidence that MI was effective in this population because of the use of reflective listening, empathy, acknowledgement of cultural beliefs, effort to overcame barriers and constraints, facilitating an action plan, building skills, and activating support resources.

Despite the encouraging results shown above, the evidence to date is preliminary. Moreover, in the above studies, caregivers were rarely involved and few received help to improve their contributions to HF patient self-care. Caregivers are acknowledged as having a key role in helping HF patients improving self-care ${ }^{10}$ but given that a psychometrically sound measure of this contribution was developed only in $2013^{9}$ we do not know yet if improving this contribution with an intervention can influence HF patient self-care. Therefore, the aims of this RCT are: 1) to evaluate the effect of MI in HF patients and caregivers in improving self-care maintenance in HF patients (primary outcome); 2) to evaluate if MI in caregivers improves patient self-care over and above MI performed solely on patients; 3) to evaluate the effect of MI on the following secondary outcomes: a) in HF patients: self-care management, self-care confidence, HF somatic symptom perception, generic and disease-specific quality of life, anxiety and depression, cognition, sleep quality, mutuality with caregiver, hospitalizations, use of emergency services, and mortality; b) in caregivers: caregiver contribution to self-care, quality of life, anxiety and depression, sleep, mutuality with patient, preparedness, and social support.

\section{Methods}




\section{Study design}

This is a three-arm randomized controlled trial (Figure 1) that complies with the Declaration of Helsinki, has been approved by the Institutional Review Board of the University of Rome "Tor Vergata" and has been registered at ClinicalTrials.gov (Identifier: NCT02894502). In this trial patients will be randomized in three arms: 1) MI intervention to only patients, 2) MI intervention to patients and caregivers, 3) standard of care to patients and caregivers.

\section{Intervention}

MI will be delivered by registered nurses who have attended a 40-hour course on MI. This intervention will be performed in Arm 1 to only patients and in Arm 2, to both patients and caregivers. The intervention will include a first session (about 60 minutes) where the interventionist will address one or two aspects of self-care that the participants want to address. Guided by the principles of MI, the interventionist will develop discrepancy (e.g., helping the patient/caregiver to see that current behaviors would impede the ability to reach health goals), express empathy (e.g., with active listening and an attitude of acceptance), avoid arguing and direct confrontation (e.g., being respectful of patient/caregiver choices or preferences), roll with resistance (e.g., by involving patient and caregiver in problem solving) and support self-efficacy and optimism (e.g., by verbal persuasion and encouraging a focus on past successes). After this first intervention, the same interventionist will contact the participant by telephone to bolster the first intervention and provide further support as needed. These telephone contacts will be done three times at two week intervals following the first intervention (for a total of two months). Patients and caregivers that receive the intervention also will be given informational material on HF management that is consistent with international guidelines.

\section{Treatment fidelity}


All interventions will be audio recorded in order to assess the quality of MI for treatment fidelity purposes as described in the Motivational Interviewing Treatment Integrity Coding Manual $4.1 .^{25}$

Control group

Patients and caregivers in the control group (Arm 3) will receive the standard care, which in Italy generally includes oral information on the disease and its treatment given to patients and their family members and a medical check-up every 6-12 months depending on patient condition. The control group also will be given the same informational material given to the intervention groups in arms 1 and 2.

Recruitment and eligibility assessment of study participants

HF patients and caregivers will be recruited in several hospital, outpatient, and community settings across Italy. HF patients and caregivers will be assessed for study eligibility based on the following inclusion and exclusion criteria. The inclusion criteria for patients are: 1) a confirmed diagnosis of HF according to international guidelines; ; ; 2) New York Heart Association (NYHA) functional class II- IV; 3) inadequate self-care assessed with a score of 0, 1 or 2 in at least two items of the self-care maintenance or self-care management scales of the Self-Care Heart Failure Index (SCHFI); 3) willingness to participate in the study and to sign the informed consent form. The exclusion criteria for patients are: 1) severe cognitive impairment evaluated with a score $0-4$ on the Six-item screener ${ }^{26}$; 2) acute coronary syndrome event during the last three months; 4) living in a residential settings (e.g., nursing home): 5) caregiver not willing to participate in the study.

Inclusion criteria for caregivers are: 1) designated by the patient as the primary informal caregiver, that is, the person inside or outside the family who takes most of care of the HF patient; 2) Exclusion criteria for caregiver are: 1) patient not willing to participate in the study. 
Patients' and caregivers' sociodemographic characteristics will be assessed at baseline. At baseline patients also will be assessed for their HF clinical characteristics (e.g., NYHA functional class), comorbidity with the Charlson Comorbidity Index, ${ }^{27}$ and cognition, with the Montreal Cognitive Assessment. ${ }^{28}$ Follow-up assessment will be performed at 3, 6, 9 and 12 months after enrolment, patients and caregivers will be tested with a battery of psychometrically sound tools (Table 1) in order to evaluate the primary and secondary outcomes. Baseline and follow-up assessment will be performed by trained nurse research assistants, who are blinded to group and different than those who perform MI.

\section{Randomization and blinding}

After enrolment and baseline data collection, each patient-caregiver dyad will be randomized (1:1:1) to one of the three arms. Randomization will be done at the University of Rome Tor Vergata with the use of an informatics software program that will generate randomization lists.

\section{Outcome measures}

\section{Primary outcome}

The primary outcome of this RCT will be HF self-care maintenance in patients that will be measured with the Self-Care Maintenance Scale of the Self-Care of HF Index version 6.2 (SCHFI). ${ }^{29}$ The SCHFI is an instrument used worldwide to measure the self-care dimensions of maintenance and management. The Self-Care Maintenance Scale captures HF symptom monitoring (e.g., weighting every day) and treatment adherence (e.g., taking medications as prescribed) and was tested for its validity and reliability ${ }^{29}$. The Self-Care Maintenance Scale yields a score from 0 to 100 with higher scores meaning better self-care maintenance. The primary outcome of self-care maintenance of patients will be evaluated 3-months from the enrolment. Also, we will evaluate HF patient self-care maintenance at 6,9 and 12 months from the enrolment. 
Several secondary outcomes will be evaluated with a battery of tools, all with established validity and reliability (Table 1). Specifically, in patients we will use: the Self-Care Management and Self-Care Confidence scales of the $\mathrm{SCHFI}^{29}$ to measure the responses to symptoms and signs of HF exacerbation and the confidence in managing all self-care processes, respectively; the HF somatic perception scale ${ }^{30}$ to measure the burden of symptoms; the SF-12, ${ }^{31}$ to measure generic physical and mental QOL; the Kansas City Cardiomyopathy Questionnaire ${ }^{32}$ to measures HF specific QOL; the Hospital Anxiety and Depression Scale ${ }^{33}$ to measure anxiety and depression; the Montreal Cognitive Assessment ${ }^{28}$ to measure cognition; the Pittsburg Sleep Quality Index ${ }^{34}$ to measure sleep quality; the Mutuality scale-patient version ${ }^{35}$ to evaluate the relationship between the patient and caregiver. In caregivers we will use: the Caregiver Contribution to Self-Care of HF Index (CC-SCHFI $)^{9}$, that investigates the extent to which caregivers recommend to patients to perform self-care or perform self-care on behalf of the patients if they are unable to do so; the SF12; the Hospital Anxiety and Depression Scale; ${ }^{33}$ the Pittsburgh Sleep Quality Index; ${ }^{34}$ the Mutuality scale-caregiver version to evaluate the relationship with the patient; ${ }^{36}$ the Caregiver Preparedness Scale,${ }^{37}$ which measures caregiver preparedness to meet the patient's physical and psychological needs; the Multidimensional Scale of Perceived Social support. ${ }^{38}$ At each follow-up, caregivers will be asked about patient hospitalization, use of emergency services and death. All secondary outcomes will be evaluated baseline and 3, 6, 9 and 12 months after the enrollment (Table 1).

\section{Statistical analysis}

Sample size: A total sample of 240 subjects ( 80 per each group) achieves $90 \%$ power to detect a $8 \%$ difference in self-care maintenance ${ }^{39}$ obtained by patients at 3 months with MI intervention (arms 1 and 2, with a mean self-care maintenance of 63) versus patients in standard care (arm 3 with a mean self-care maintenance of 55) using an F test with a 0.05 significance level (one-way ANOVA). The common standard deviation within a group is assumed to be $18 .^{20}$ The sample of 240 subjects will 
also provide $83 \%$ power to detect a $8 \%$ difference in patient self-care management and self-care confidence at 3 months in arm 1 and 2 versus 3, assuming a standard deviation of $20 .{ }^{39}$ Based on the available literature and in order to account for an estimated $50 \%$ attrition rate ${ }^{20}, 480$ participants (240 patients and 240 caregivers) will be recruited. As far as the evaluation of MI in caregivers (aim 2), group sample sizes of 80 achieve $80 \%$ power to detect a difference of 8 points of caregiver selfcare maintenance and with estimated group standard deviations of 18 and with a significance level of 0.05 using a two-sided two-sample t-test. As for patient self-care management and self-care confidence, with an expected group standard deviations of 20 , the power for aim 2 is $71 \%$.

Planned statistical analysis: Measures of central tendency and of variability will be used to describe HF patient and caregiver characteristics as well as the outcome measure scores. For the primary end-point a one-way ANOVA will be used comparing arm 1 and 2 with arm 3. A two-sample t-test will be used to compare self-care of participants in arm 1 and 2 to evaluate $\mathrm{MI}$ in caregivers. The percentage of patients with a SCHFI score over $70^{39}$ will be compared with the Chi-square test. A longitudinal linear regression model will be used to evaluate the trend of Self-Care in the three arms. Secondary outcomes will be evaluated with ANOVA.

\section{Discussion}

The aim of this RCT is to evaluate the effect of MI in improving self-care maintenance in HF patients. Also, this study will allow us to see if MI has an effect on other secondary variables, such as, in patients: self-care management, self-care confidence, symptom perception, quality of life, anxiety and depression, cognition, sleep quality, mutuality with caregiver, hospitalizations, use of emergency services, and mortality; in caregivers: caregiver contribution to self-care, quality of life, anxiety and depression, sleep, mutuality with patient, preparedness, and social support.

Self-care may improve patient outcomes ${ }^{5}$ but investigators have found that self-care is low in several populations ${ }^{11,40,41}$. Despite well designed studies conducted to improve self-care, these studies have not always had good results. However, a recent systematic review ${ }^{15}$ and mixed method 
study $^{24}$ improved our understanding of the mechanisms through which programs actually work to improve HF self-care. In this systematic review, it was shown that interventions to improve HF patient self-care may be more effective if a good supportive relationship is established between the patient and the provider, if the interventions focus on self-efficacy and increasing understanding of the disease, involve other supportive people, and are individualized. In the mixed method study, it emerged that the development of discrepancy and self-efficacy were the main mechanisms involved in changes in self-care. All the above aspects are emphasized in motivational interviewing.

Several studies conducted in other patient populations ${ }^{18}$ have demonstrated that MI can be effective in facilitating behavioral change, but, to our knowledge, in HF only 5 studies with small samples have been published ${ }^{21-25}$. These studies have been promising, with improvements in selfcare maintenance, self-care confidence, quality of life and hospital readmission. Differently from other studies so far conducted with MI, in our study we will also use MI with the caregivers since they have a key role in contributing to HF care. ${ }^{10}$ Also, differently from other studies using MI, in our secondary outcomes, we will follow-up patients' self-care and caregivers' contributions to selfcare over one year, so that we can see if MI has a long lasting effect and, if so, on which variables. In this way we can also evaluate the "dose" of MI in order to personalize future interventions. For example, based on the trajectory of self-care and caregiver contribution to self-care, we can see when MI is needed in order to keep self-care sufficiently high. We demonstrated in prior work ${ }^{42}$ that, in order to significantly reduce mortality and rehospitalizations, self-care maintenance, management and confidence scores should be around a standardized score of $70(0-100$ possible range). These scores are not easy to reach since in most of studies the self-care maintenance, management and confidence scores were approximately $50 .{ }^{43}$

It has been suggested that an MI intervention could be expensive for health-care organizations to provide, since nurses spend a lot of time providing the intervention. Masterson Creber et al and Riegel et $\mathrm{al}^{21}$ have shown that a brief MI session can improve self-care 
maintenance and can reduce hospitalizations at three months from the intervention. Even though these investigators have seen the effect of MI in a short period of time, their intervention was potentially cost effective since, as we will do in this study, they performed only one session with MI and three follow-up calls to bolster the intervention.

\section{Conclusion}

HF is a debilitating illness anticipated to increase in prevalence in the future because the aging of the population. It was shown that around the $7 \%$ of the total health-care budget are for HF care in developed countries. ${ }^{44}$ As the population is aging, we can anticipate that health-care expenditures for HF will only rise in the future. Self-care can improve patients' condition and reduce readmissions but improving self-care is not easy. This study will determine if MI could help HF patients and their caregivers to improve their self-care sufficiently to reduce the burden of the illness. The next step will be to determine if this intervention is also cost-effective.

\section{Funding}

This study was funded by the Center of Excellence for Nursing Scholarship, Rome, Italy

\section{Acknowledgment}

We thank the Italian Association of Heart Failure for their support in this study.

\section{Conflict of interest}

None 
Table 1. Variables and instruments

\begin{tabular}{|c|c|c|c|c|c|c|c|}
\hline \multirow[t]{2}{*}{ Variable } & \multirow[t]{2}{*}{ Instrument } & \multirow[t]{2}{*}{ Administered to } & \multicolumn{5}{|c|}{ Times of data collection } \\
\hline & & & Baseline & 3 months & 6 month & 9 months & 12 months \\
\hline $\begin{array}{l}\text { Self-care } \\
\text { maintenance, } \\
\text { management and } \\
\text { confidence }\end{array}$ & SCHFI & $\mathrm{P}$ & $\mathrm{x}$ & $\mathrm{x}$ & $\mathrm{x}$ & $\mathrm{x}$ & $\mathrm{x}$ \\
\hline $\begin{array}{l}\text { Caregiver } \\
\text { contribution to HF } \\
\text { self-care }\end{array}$ & CC-SCHFI & $\mathrm{C}$ & $\mathrm{x}$ & $\mathrm{x}$ & $\mathrm{x}$ & $\mathrm{x}$ & $\mathrm{x}$ \\
\hline HF symptoms & HFSPS & $\mathrm{P}$ & $\mathrm{x}$ & $\mathrm{x}$ & $\mathrm{x}$ & $\mathrm{x}$ & $\mathrm{x}$ \\
\hline Generic QOL & SF-12 & $\mathrm{P}-\mathrm{C}$ & $\mathrm{x}$ & $\mathrm{x}$ & $\mathrm{x}$ & $\mathrm{x}$ & $\mathrm{x}$ \\
\hline Specific QOL & $\mathrm{KCCQ}$ & $\mathrm{P}$ & $\mathrm{x}$ & $\mathrm{x}$ & $\mathrm{x}$ & $\mathrm{x}$ & $\mathrm{x}$ \\
\hline Anxiety/Depression & HADS & $\mathrm{P}-\mathrm{C}$ & $\mathrm{x}$ & $\mathrm{x}$ & $\mathrm{x}$ & $\mathrm{x}$ & $\mathrm{x}$ \\
\hline Cognition & MOCA & $\mathrm{P}$ & $\mathrm{x}$ & & & & \\
\hline $\begin{array}{l}\text { Caregiver } \\
\text { Preparedness }\end{array}$ & CPS & $\mathrm{C}$ & $\mathrm{x}$ & $\mathrm{x}$ & $\mathrm{x}$ & $\mathrm{x}$ & $\mathrm{x}$ \\
\hline Social support & MSPSS & $\mathrm{C}$ & $\mathrm{x}$ & $\mathrm{x}$ & $\mathrm{x}$ & $\mathrm{x}$ & $\mathrm{X}$ \\
\hline Hospitalizations & Questionnaire & $\mathrm{C}$ & & $\mathrm{x}$ & $\mathrm{x}$ & $\mathrm{x}$ & $\mathrm{x}$ \\
\hline $\begin{array}{l}\text { Use of emergency } \\
\text { services }\end{array}$ & Questionnaire & $\mathrm{C}$ & & $\mathrm{x}$ & $\mathrm{x}$ & $\mathrm{x}$ & $\mathrm{x}$ \\
\hline Mortality & Questionnaire & $\mathrm{C}$ & & $\mathrm{x}$ & $\mathrm{x}$ & $\mathrm{x}$ & $\mathrm{x}$ \\
\hline
\end{tabular}

Note. $\mathrm{P}=$ patient; $\mathrm{C}=$ caregiver; $\mathrm{SCHFI}=$ Self-Care of Heart Failure Index; CC-SCHFI = Caregiver contribution to self-care of HF index; HFSPS = Heart Failure Somatic Perception Scale; QOL = quality of life; SF-12 = Short Form 12; KCCQ = Kansas City Cardiomyopathy Questionnaire; HADS = Hospital Anxiety and Depression Scale; MOCA = Montreal Cognitive Assessment; PSQI $=$ Pittsburgh Sleep Quality Index; MS = Mutuality scale; CPS = Caregiver preparedness scale; MSPSS = Multidimensional Scale of Perceived Social Support. 
Figure 1. Flow-chart of the study

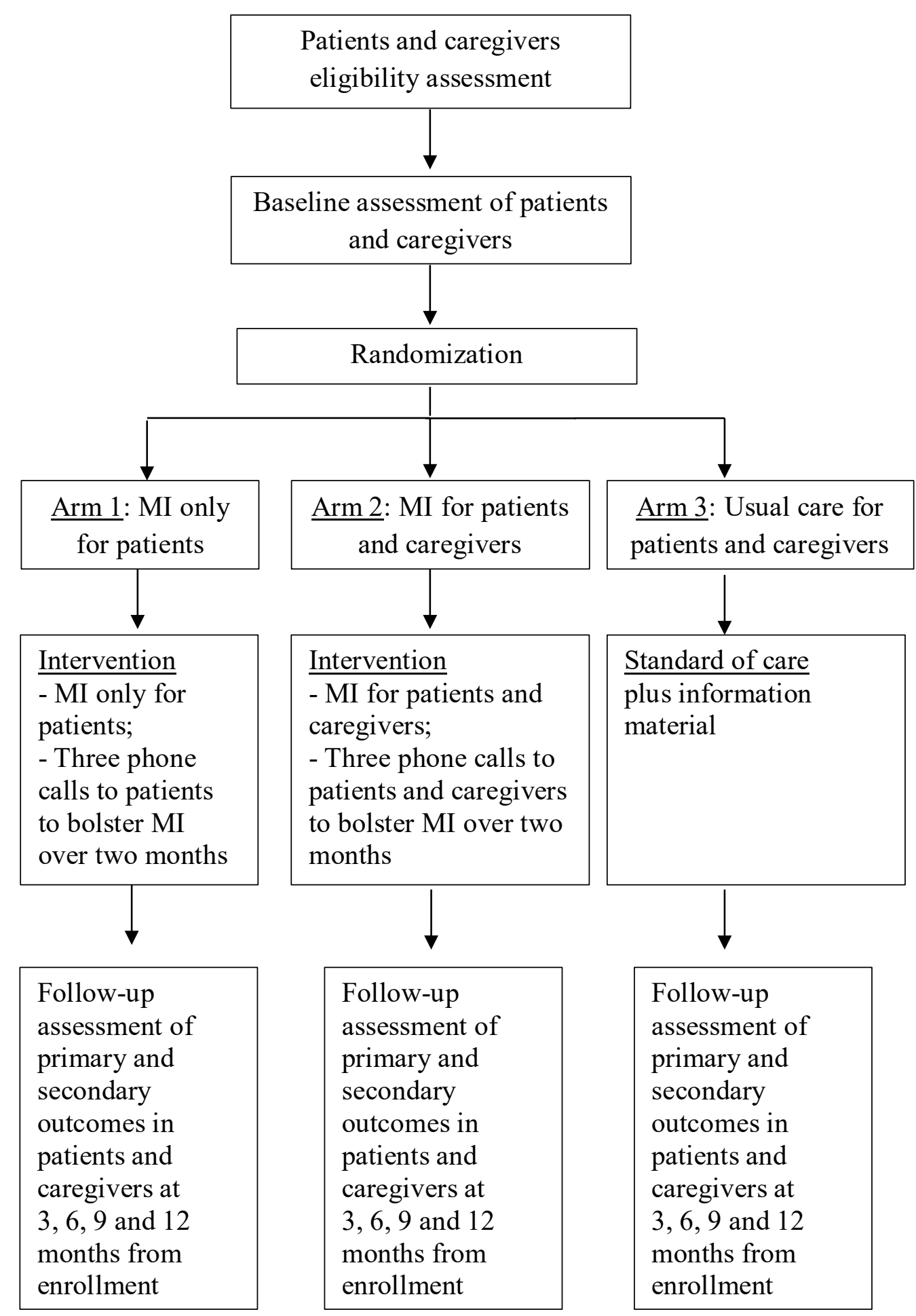




\section{References}

1. Ponikowski P, Voors AA, Anker SD, et al. 2016 ESC Guidelines for the diagnosis and treatment of acute and chronic heart failure: The Task Force for the diagnosis and treatment of acute and chronic heart failure of the European Society of Cardiology (ESC)Developed with the special contribution of. Eur Heart J. May 2016. doi:10.1093/eurheartj/ehw128.

2. Mozaffarian D, Benjamin EJ, Go AS, et al. Heart disease and stroke statistics--2015 update: a report from the American Heart Association. Circulation. 2015;131(4):e29-322. doi:10.1161/CIR.0000000000000152CIR.0000000000000152 [pii].

3. Bekelman DB, Rumsfeld JS, Havranek EP, et al. Symptom burden, depression, and spiritual well-being: a comparison of heart failure and advanced cancer patients. J Gen Intern Med. 2009;24(5):592-598. doi:10.1007/s11606-009-0931-y.

4. Davidson PM, Abernethy AP, Newton PJ, Clark K, Currow DC. The caregiving perspective in heart failure: a population based study. BMC Health Serv Res. 2013;13:342. doi:10.1186/1472-6963-13-342.

5. Smith CE, Piamjariyakul U, Wick JA, et al. Multidisciplinary group clinic appointments: the Self-Management and Care of Heart Failure (SMAC-HF) trial. Circ Hear Fail. 2014;7(6):888-894. doi:10.1161/CIRCHEARTFAILURE.113.001246.

6. Riegel B, Dickson V V, Faulkner KM. The Situation-Specific Theory of Heart Failure SelfCare: Revised and Updated. J Cardiovasc Nurs. 2015. doi:10.1097/JCN.0000000000000244.

7. Buck HG, Dickson VV, Fida R, et al. Predictors of hospitalization and quality of life in heart failure: A model of comorbidity, self-efficacy and self-care. Int J Nurs Stud. 2015;52(11):1714-1722. doi:10.1016/j.ijnurstu.2015.06.018.

8. Vellone E, Riegel B, D'Agostino F, et al. Structural equation model testing the situation- 
specific theory of heart failure self-care. $J$ Adv Nurs. 2013;69(11):2481-2492.

9. Vellone E, Riegel B, Cocchieri A, et al. Validity and reliability of the caregiver contribution to self-care of heart failure index. J Cardiovasc Nurs. 2013;28(3):245-255.

http://www.ncbi.nlm.nih.gov/pubmed/22760172.

10. Vellone E, Dagostino F, Buck HG, et al. The key role of caregiver confidence in the caregivers contribution to self-care in adults with heart failure. Eur J Cardiovasc Nurs. 2015;14(5):372-381. doi:10.1177/1474515114547649.

11. Jaarsma T, Strömberg A, Ben Gal T, et al. Comparison of self-care behaviors of heart failure patients in 15 countries worldwide. Patient Educ Couns. 2013;92(1):114-120. doi:10.1016/j.pec.2013.02.017.

12. Freedland KE, Carney RM, Rich MW, Steinmeyer BC, Rubin EH. Cognitive Behavior Therapy for Depression and Self-Care in Heart Failure Patients: A Randomized Clinical Trial. JAMA Intern Med. 2015;63108:1-10. doi:10.1001/jamainternmed.2015.5220.

13. Dracup K, Moser DK, Pelter MM, et al. Randomized, controlled trial to improve self-care in patients with heart failure living in rural areas. Circulation. 2014;130(3):256-264. doi:10.1161/CIRCULATIONAHA.113.003542.

14. Jonkman NH, Westland H, Groenwold RHH, et al. What Are Effective Program Characteristics of Self-Management Interventions in Patients With Heart Failure? An Individual Patient Data Meta-analysis. J Card Fail. 2016;0(0):1137-1146. doi:10.1016/j.cardfail.2016.06.422.

15. Clark AM, Wiens K, Banner D, et al. A systematic review of the main mechanisms of heart failure disease management interventions. Heart. 2016:heartjnl-2015-308551. doi:10.1136/heartjnl-2015-308551. 
16. Miller WR, Rollnick S. Ten Things that Motivational Interviewing Is Not. Behav Cogn Psychother. 2009;37(2):129. doi:10.1017/S1352465809005128.

17. Palacio A, Garay D, Langer B, Taylor J, Wood BA, Tamariz L. Motivational Interviewing Improves Medication Adherence: a Systematic Review and Meta-analysis. J Gen Intern Med. 2016;31(8):929-940. doi:10.1007/s11606-016-3685-3.

18. Ekong G, Kavookjian J, Zhuo X, et al. Motivational interviewing and outcomes in adults with type 2 diabetes: A systematic review. Patient Educ Couns. 2016;99(6):944-952. doi:10.1016/j.pec.2015.11.022.

19. Lee WWM, Choi KC, Yum RWY, et al. Effectiveness of motivational interviewing on lifestyle modification and health outcomes of clients at risk or diagnosed with cardiovascular diseases: A systematic review. Int J Nurs Stud. 2016;53:331-341. doi:10.1016/j.ijnurstu.2015.09.010.

20. Masterson Creber R, Patey M, Lee CS, Kuan A, Jurgens C, Riegel B. Motivational interviewing to improve self-care for patients with chronic heart failure: MITI-HF randomized controlled trial. Patient Educ Couns. 2015. doi:10.1016/j.pec.2015.08.031.

21. Riegel B, Masterson Creber R, Hill J, Chittams J, Hoke L. Effectiveness of Motivational Interviewing in Decreasing Hospital Readmission in Adults With Heart Failure and Multimorbidity. Clin Nurs Res. 2016;25(4):362-377. doi:10.1177/1054773815623252.

22. Paradis V, Cossette S, Frasure-Smith N, Heppell S, Guertin M-C. The efficacy of a motivational nursing intervention based on the stages of change on self-care in heart failure patients. J Cardiovasc Nurs. 2010;25(2):130-141. doi:10.1097/JCN.0b013e3181c52497.

23. Brodie DA, Inoue A, Shaw DG. Motivational interviewing to change quality of life for people with chronic heart failure: a randomised controlled trial. Int J Nurs Stud. 
2008;45(4):489-500. doi:10.1016/j.ijnurstu.2006.11.009.

24. Riegel B, Dickson VV, Garcia LE, Creber RM, Streur M. Mechanisms of change in self-care in adults with heart failure receiving a tailored, motivational interviewing intervention. Patient Educ Couns. 2016:6-11. doi://dx.doi.org/10.1016/j.pec.2016.08.030.

25. Moyers T, Manuel J, Ernst D. Motivational Interviewing Treatment Integrity Manual 4.1. Unpublished manual; 2014.

26. Callahan CM, Unverzagt FW, Hui SL, Perkins AJ, Hendrie HC. Six-item screener to identify cognitive impairment among potential subjects for clinical research. Med Care. 2002;40(9):771-781. doi:10.1097/01.MLR.0000024610.33213.C8.

27. Charlson M, Pompei P, Ales K, MacKenzie C. A new method of classifying prognostic comorbidity in longitudinal studies: Development and validation. $J$ Chronic Dis. $1987 ; 40: 373-383$.

28. Nasreddine ZS, Phillips NA, Bédirian V, et al. The Montreal Cognitive Assessment, MoCA: a brief screening tool for mild cognitive impairment. J Am Geriatr Soc. 2005;53(4):695-699. doi:10.1111/j.1532-5415.2005.53221.x.

29. Vellone E, Riegel B, Cocchieri A, et al. Psychometric properties of the self-care of heart failure index version 6.2. Res Nurs Heal. 2013;36(5):500-511. doi:10.1002/nur.21554.

30. Jurgens C, Lee C, Riegel B. Psychometric Analysis of the Heart Failure Somatic Perception Scale as a Measure of Patient Symptom Perception. J Cardiovasc .... 2015;0(0):1-8. doi:10.1097/JCN.0000000000000320.

31. Ware Jr. J, Kosinski M, Keller SD. A 12-Item Short-Form Health Survey: construction of scales and preliminary tests of reliability and validity. Med Care. 1996;34(3):220-233. http://www.ncbi.nlm.nih.gov/pubmed/8628042. 
32. Green CP, Porter CB, Bresnahan DR, Spertus JA. Development and evaluation of the Kansas City Cardiomyopathy Questionnaire: a new health status measure for heart failure. J Am Coll Cardiol. 2000;35(5):1245-1255. doi:10.1016/S0735-1097(00)00531-3.

33. Zigmond AS, Snaith RP. The Hospital Anxiety and Depression Scale. Acta Psychiatr Scand. $1983 ; 67: 361-370$.

34. Buysse DJ, Reynolds CF, Monk TH, et al. The Pittsburgh Sleep Quality Index: a new instrument for psychiatric practice and research. Psychiatry Res. 1989;28(2):193-213. doi:10.1016/0165-1781(89)90047-4.

35. Simeone S, Alvaro R, Cohen MZ, Vellone E, Savini S, Pucciarelli G. The lived experiences of stroke caregivers three months after discharge of patients from rehabilitation hospitals. Prof Inferm. 2016;69(2):103-112.

36. Archbold PG, Stewart BJ, Greenlick MR, Harvath T. Mutuality and preparedness as predictors of caregiver role strain. Res Nurs Health. 1990;13(6):375-384. http://www.ncbi.nlm.nih.gov/pubmed/2270302. Accessed August 3, 2016.

37. Savini S, Buck HG, Dickson VV, et al. Quality of life in stroke survivor-caregiver dyads: A new conceptual framework and longitudinal study protocol. $J$ Adv Nurs. 2015;71(3):676-687. doi:10.1111/jan.12524.

38. Zimet GD, Powell SS, Farley GK, Werkman S, Berkoff KA. Psychometric characteristics of the Multidimensional Scale of Perceived Social Support. J Pers Assess. 1990;55(3-4):610617. doi:10.1080/00223891.1990.9674095.

39. Riegel B, Lee CS, Dickson V V, Carlson B. An update on the self-care of heart failure index. J Cardiovasc Nurs. 2009;24(6):485-497. doi:10.1097/JCN.0b013e3181b4baa0.

40. Ausili D, Masotto M, Dall'Ora C, Salvini L, Di Mauro S. A literature review on self-care of 
chronic illness: definition, assessment and related outcomes. Prof Inferm. 2014;67(3):180189. doi:10.7429/pi.2014.673180.

41. Ausili D, Rebora P, Mauro S Di, et al. Clinical and socio-demographic determinants of selfcare behaviours in patients with heart failure and diabetes mellitus: a multicentre crosssectional study. Int J Nurs Stud. 2016;63:18-27. doi:10.1016/j.ijnurstu.2016.08.006.

42. Vellone E, Cocchieri A, Paturzo M, et al. Poor heart failure symptom response is associated with an increased risk of death and emergent healthcare utilization. Eur Heart J. 2016;37:1340.

43. Wu J-R, Lennie TA, Moser DK. A prospective, observational study to explore health disparities in patients with heart failure-ethnicity and financial status. Eur J Cardiovasc Nurs. March 2016. doi:10.1177/1474515116641296.

44. Farré N, Vela E, Clèries M, et al. Medical resource use and expenditure in patients with chronic heart failure: a population-based analysis of 88195 patients. Eur J Heart Fail. 2016;18(9):1132-1140. doi:10.1002/ejhf.549. 